\title{
Tutorías entre pares: una oportunidad de formación para futuros profesores de matemáticas ${ }^{1}$
}

\author{
Peer tutoring: training opportunity for future math teachers \\ Tutoria entre pares: oportunidade de formação \\ para professores de matemática futuros
}

Recibido: mayo de 2013

Aceptado: agosto de 2013
Islenis Carolina Botello C. ${ }^{2}$ Sandra Evely Parada R. ${ }^{3}$

\section{Resumen}

En este documento presentamos algunos resultados de una investigación cualitativa que se ha llevado a cabo tras el diseño e implementación de un programa de tutorías entre pares, facilitadas por profesores en formación y coordinada por formadores de profesores. Dicha investigación (aún en curso) tiene entre sus propósitos conceptualizar los aprendizajes emergentes de los profesores en formación -tutores- dentro de procesos de seguimiento y acompañamiento académico "especializado. Al llevar una primera etapa de la implementación, se ha encontrado que las tutorías entre pares sirven como escenario potencial para la práctica real de los profesores en formación.

Palabras clave: Tutoría entre pares; profesor; formación inicial de profesores; Matemáticas escolares; cálculo; cálculo diferencial.

\begin{abstract}
In this paper we present some results of a qualitative research that has been carried out after the design and implementation of a peer tutoring program, facilitated by teachers in training, coordinated by teacher trainers. The investigation (still in progress) has among its purposes conceptualize emerging learning student teachers-tutors-in monitoring processes and academic support "specialized. By taking the first stage of implementation, found that peer tutoring serve as potential scenario for the actual practice of student teachers.
\end{abstract}

Keywords: peer tutoring, teacher, initial teacher training, school mathematics, calculus, differential calculus.

1 Artíulo de Investigación

2 Universidad Industrial de Santander, Maestría en Educación Matemática. Contacto: islenis.botello@correo.uis.edu.co

3 Universidad Industrial de Santander. Contacto: sparada@matematicas.uis.edu.co 


\section{Resumo}

Neste artigo apresentamos alguns resultados de uma pesquisa qualitativa que tem sido realizadas após a concepção e implementação de um programa de tutoria entre pares, facilitado pelos professores em formação, coordenada por formadores de professores. A investigação (ainda em andamento) tem entre seus objetivos conceituar emergente aprendizagem dos alunos professores-tutores em processos de acompanhamento e de apoio acadêmico "especializado. Ao tomar a primeira fase de implementação, constatou que a tutoria de pares servir como cenário potencial para a prática de futuros professores.

Palavras-chave: tutoria de pares, o professor, a formação inicial de professores, matemática escolar, cálculo, cálculo diferencial.

\section{Introducción}

En la Universidad Industrial de Santander (UIS) se ha presenciado una situación desde hace varios años, donde los estudiantes de primeros niveles presentan altos índices de fracaso académico y deserción; una de las asignaturas donde mayor índice presenta esta tasa, es cálculo diferencial. Para ello, se han creado alternativas que tratan de subsanar de una u otra forma dicha situación, con las cuales no se ha logrado generar un impacto relevante.

De otro lado, la UIS cuenta con un programa de Licenciatura en Matemáticas, la cual forma los profesores en matemáticas de la región. En su plan de estudios, la práctica docente se realiza en los dos últimos semestres, de allí que hemos observado como profesoras de la UIS (y egresadas de la misma), una articulación distante entre lo que se van aprendiendo teóricamente (en los cursos de formación matemática y didáctica) y la práctica real con la que se van a enfrentar en sus salones de clase. De aquí surge nuestra investigación, la cual desde el diseño de una alternativa curricular que puede, por un lado, atender las necesidades (conceptuales o procedimentales) de los estudiantes en el cálculo diferencial, y por el otro, permitir a los profesores en formación tener un primer acercamiento a la práctica pedagógica docente.

Al plantear la alternativa anteriormente mencionada, surge la siguiente pregunta: ¿cómo diseñar y guiar procesos de acompañamiento y seguimiento académico (tutorías) a los estudiantes que presentan dificultades en el aprendizaje de cálculo diferencial posibilitadas por profesores en formación?

Aspectos teóricos y revisión bibliográfica

Para responder a nuestra pregunta de investigación, necesitamos definir algunos elementos teóricos que usaremos tanto en el diseño metodológico de la investigación, como en la delimitación del programa de seguimiento y acompañamiento.

\section{Tutorías entre pares.}

Según Goodlad \& Hirst (1989) se entiende por tutoría entre pares como el conjunto de prácticas, en las cuales algunos estudiantes ayudan a otros estudiantes, y aprenden enseñando. Luego, existe un tutor (quien ayuda) y un estudiante tutorizado (el alumno que presenta dificultades en su proceso de aprendizaje). De acuerdo a Miranda (2010) y Cardozo (2011), este tipo de tutorías se fundamenta en la mayor aproximación empática que el estudiante tutorado puede encontrar en los tutores próximos en edad y, con problemáticas semejantes.

Pensamiento matemático, y Pensamiento pedagógico y didáctico del profesor de matemáticas

Para efectos de nuestra investigación no hablaremos de conocimientos ni saberes, sino en términos de pensamientos, como lo propone Parada (2011) quien menciona que el pensamiento reflexivo del profesor de matemática puede revisarse desde tres componentes: el matemático, el pedagógico y 
didáctico, y el orquestal. No obstante, para nuestra investigación contemplamos los dos primeros componentes [ya que el tercero subyace en el marco de un aula de clase, contrario a lo que planteamos en las tutorías], estos componentes se definen así:

Pensamiento matemático. Resulta de la necesidad del profesor para hacer uso de sus conocimientos del contenido matemático escolar en el desarrollo de sus prácticas profesionales: a) proponer tareas; b) seleccionar, usar y diseñar recursos; c) comunicarse en el aula; d) hacer adaptaciones curriculares; e) evaluar; f) colaborar; y g) profesionalizarse.

Pensamiento pedagógico y didáctico. Se da cuando el profesor se cuestiona sobre las diferentes maneras de acercar los conocimientos matemáticos a los estudiantes, buscando las formas más útiles de representar los contenidos mediante analogías, ilustraciones, ejemplos, explicaciones, y demostraciones que permitan hacerla más comprensible a los alumnos.

Es precisamente en el desarrollo de estos dos pensamientos que consideramos que las tutorías entre pares pueden ser una oportunidad de formación temprana de los profesores de matemáticas, pues es en la práctica misma donde se puede comprender la complejidad de la práctica pedagógica, en nuestro caso de la educación matemática.

\section{Metodología}

El tipo de investigación que se está realizando es exploratoria-descriptiva, ya que al indagarse en la literatura se encontró diferentes estudios sobre procesos de seguimiento y acompañamiento (tutorías), de los cuales pocos estaban dirigidos al Cálculo Diferencial o pocos emplean las tutorías como puente entre la práctica ficticia de un profesor en formación y la práctica pedagógica real. El trabajo de campo de nuestra investigación fue de un año y tuvo como población a los estudiantes de la asignatura de Didáctica del Cálculo, quienes fungieron como tutores. Esta asignatura tiene entre sus propósitos ofrecer -desde la teoría y la práctica- fundamentos para el diseño de metodologías adecuadas para el aprendizaje del cálculo. Para el diseño, recolección de información, análisis de datos, ejecución, y posterior evaluación de la alternativa se desarrollaron las siguientes fases:

Fase 0 . Un primer acercamiento. En esta fase se hizo un estudio de los programas de seguimiento a estudiantes en la universidad, donde se hace la descripción del fenómeno de estudio para establecer un punto de partida. También se indagó sobre este tipo de programas en otras instituciones nacionales y extranjeras.

Fase I. Diseño de la alternativa. A partir del estudio realizado en la fase anterior, se diseñó un programa de seguimiento y acompañamiento a estudiantes, específicamente para Cálculo I de la UIS; esto a partir de la revisión de la literatura acerca de programas existentes en otras universidades y en la UIS.

Fase II. Primera implementación del programa. Esta fase se buscaba observar la relación de tutorías entre pares a: alumnos-docentes y estudiantes de cálculo. A partir de las fases anteriores se implementa por primera vez el programa. En esta fase se analizaron relaciones como: experiencia del estudiante de Licenciatura en Matemáticas como tutor, y receptividad por parte del estudiante de cálculo I. También se aprovechó para considerar elementos organizacionales del programa. Esta fase se realizó en el primer semestre de 2012 y contó con la participación de 64 estudiantes aproximadamente.

Inicialmente los estudiantes fueron seleccionados a partir de una prueba diagnóstica y se distribuyeron 3 estudiantes por tutor. Posteriormente, los profesores decidían cuáles estudiantes continuaban o ingresaban a la prueba piloto, acorde a su desempeño académico. Tanto los alumnos como los tutores eran supervisados por formadores de profesores (las autoras y el profesor titular del curso de Didáctica del Cálculo).

Fase III. Rediseño del programa. Ya obtenidos los primeros datos recolectados en la Fase II se analizarán estos datos para efectuar un rediseño de la alternativa curricular. A partir de los resultados de la primera implementación se hicieron algunos 
cambios dentro de las tutorías, entre ellos: se aumentó el número de horas de las tutorías ( 1 a 2 horas semanales), algunos tutores sólo trabajaban con un estudiante, se les brindó una corta inducción a los tutores, se dispuso de tres distintos horarios para ajustarse tanto al horario del tutor como el del estudiante. La mayoría de ajustes fueron concernientes a la logística del proceso.

Fase IV. Implementación formal del programa. Obtenidos los resultados y realizado su posterior análisis, se consiguió los datos relevantes para conformar la alternativa, se pone formalmente en escena del proceso de acompañamiento y seguimiento a estudiantes de Cálculo I en la UIS. La nueva implementación se realizó en el segundo semestre académico de 2012, donde las tutorías entre pares tienen como objetivo intervenir en el proceso de enseñanza-aprendizaje del cálculo diferencial en su alumno-beneficiario (estudiante tutorizado), mediante las actividades que ha diseñado él (tutor), buscando coadyuvar en las dificultades que presenta el estudiante, a partir de adaptaciones curriculares al programa que tiene la asignatura de Cálculo Diferencial.

Fase V. Análisis de los datos de la Fase IV. De un primer análisis ha surgido como un primer resultado la identificación de los perfiles de los profesores de matemáticas en formación, dichos perfiles son: i) fortalezas en el pensamiento matemático $(\mathrm{PM})$ y fortalezas en el pensamiento pedagógico y didáctico (PPD); ii) fortalezas en el PM y debilidades en el PPD; iii) debilidades en el PM y fortalezas en el PPD, y iv) debilidades en el PM y PPD. Para responder a nuestra pregunta de investigación realizaremos un estudio de casos y para ello se selecciona un alumno-docente que represente cada perfil.

De cada uno de esos casos se están sistematizando datos como: encuestas, grabaciones de audio y video, bitácoras, formatos de tutorías e informes finales del proceso. En estos últimos los tutores dan cuenta de los avances particulares y grupales de los estudiantes a los que siguieron y acompañaron académicamente durante el semestre.

Fase VI. Planteamiento de un programa de ASAE en la UIS. Al realizar las fases anteriores y analizar los datos obtenidos en ellas, se documentará y caracterizará un programa curricular como ASAE (Atención, Seguimiento y Acompañamiento a Estudiantes) de Cálculo I en la UIS.

\section{Resultados}

En la primera implementación del programa se encontró que los estudiantes del curso de Cálculo mostraron vacíos cognitivos y bajo nivel de comprensión en temas como: números reales, ecuaciones lineales y cuadráticas, funciones, composición de funciones, desigualdades y sistemas de ecuaciones, límites, continuidad en un punto, dificultad al resolver problemas específicamente los que tenían que ver con procesos algebraicos.

Desde el pensamiento matemático, las tutorías fueron una oportunidad para que los tutores recordaran o fortalecieran los contenidos matemáticos del curso, pues en algunos casos se presentaron dificultades del dominio conceptual. Al parecer los tutores habían olvidado dichos contenidos, pues en este momento de formación estos alumnos-docentes ya han visto cuatro cursos alrededor del cálculo; esto nos lleva a reflexionar sobre las limitaciones que puede tener la tutoría entre pares y las alternativas de supervisión que deben generarse para que éstas puedan tener el impacto deseado.

Desde el pensamiento didáctico, los tutores tuvieron la oportunidad de identificar debilidades conceptuales y de métodos de estudio en sus compañeros-alumnos, a partir de allí trataron de plantear actividades para atender casos particulares. Los alumnos-docentes además diseñaron talleres en los que utilizaron lo que estaban aprendiendo en su curso de Didáctica del Cálculo, como por ejemplo el uso de programas de Geometría Dinámica, para favorecer procesos de visualización alrededor de la continuidad, el infinito, el límite, entre otras nociones del Cálculo Diferencial.

Los resultados antes expuestos refuerzan nuestra idea de que este tipo de propuestas es viable para la consolidación de la relación teoría y práctica, donde el alumno-docente cuenta con espacios dentro de la misma universidad para llevarlas a una práctica profesional. 


\section{Referencias}

Cardozo, C. (2011). Tutoría entre pares como una es trategia pedagógica universitaria. Revista educación y educadores, 14(2), 309-325.

Goodlad, S., \& Hirst, B. (1989). Peer tutoring: A gui de to learning by teaching. England: Kogan Page Ltd.

Miranda, A. (2010). Peer tutoring: aprendiendo entre estudiantes. Médica UIS. Revista de los estudiantes de medicina de la Universidad Industrial de Santander, 23, 7-8.
Parada, S. (2011). Reflexión y acción en comuni dades de práctica: Un modelo de desarrollo profesional(Tesis doctoral). Centro de investigación y estudios avanzados del Instituto Politécnico Nacional, México.

Rico, L. (2004). Reflexiones sobre la formación inicial del profesor de matemáticas de secundaria. Revista de curriculum y formación del profesorado, 8(1), 1-15. 